\title{
A NOTE ON FRACTIONAL DIFFERENTIAL SUBORDINATION BASED ON THE SRIVASTAVA-OWA FRACTIONAL OPERATOR
}

\author{
Rabha W. Ibrahim ${ }^{1}$ and Maslina Darus ${ }^{2 *}$ \\ ${ }^{1}$ Institute of Mathematical Sciences \\ Universiti Malaya, 50603, Kuala Lumpur, Malaysia \\ ${ }^{2}$ School of Mathematical Sciences, Faculty of Science and Technology \\ Universiti Kebangsaan Malaysia, Bangi 43600, Selangor Darul Ehsan, Malaysia \\ rabhaibrahim@yahoo.com, maslina@ukm.my
}

\begin{abstract}
In this work, we consider a definition for the concept of fractional differential subordination in sense of Srivastava-Owa fractional operators. By employing some types of admissible functions involving differential operator of fractional order, we illustrate applications.
\end{abstract}

Key Words- Admissible function, Fractional differential equation, Subordination, Superordination, Srivastava-Owa fractional operators, Univalent function

\section{INTRODUCTION}

Recently, the theory of fractional calculus has found interesting applications in the theory of analytic functions. The classical definitions of fractional operators and their generalizations have fruitfully been applied in obtaining, for example, the characterization properties, coefficient estimates [1], distortion inequalities [2] and convolution structures for various subclasses of analytic functions and the works in the research monographs. In [3], Srivastava and Owa gave definitions for fractional operators (derivative and integral) in the complex z-plane $\mathrm{C}$ as follows:

Definition 1.1. The fractional derivative of order $\alpha$ is defined, for a function $f(z)$ by

$$
D_{z}^{\alpha} f(z):=\frac{1}{\Gamma(1-\alpha)} \frac{d}{d z} \int_{0}^{z} \frac{f(\zeta)}{(z-\zeta)^{\alpha}} d \zeta ; 0 \leq \alpha<1,
$$

where the function $f(z)$ is analytic in simply-connected region of the complex z-plane $\mathrm{C}$ containing the origin and the multiplicity of $(z-\zeta)^{-\alpha}$ is removed by requiring $\log (z-\zeta)$ to be real when $(z-\zeta)>0$.

Definition 1.2. The fractional integral of order $\alpha$ is defined, for a function $f(z)$, by

$$
I_{z}^{\alpha} f(z):=\frac{1}{\Gamma(\alpha)} \int_{0}^{z} f(\zeta)(z-\zeta)^{\alpha-1} d \zeta ; \alpha>0,
$$

where the function $f(z)$ is analytic in simply-connected region of the complex z-plane (C) containing the origin and the multiplicity of $(z-\zeta)^{\alpha-1}$ is removed by requiring 
$\log (z-\zeta)$ to be real when $(z-\zeta)>0$.

Remark 1.1. From Definitions 1.1 and 1.2, we have

$$
D_{z}^{\alpha}\left\{z^{\mu}\right\}=\frac{\Gamma(\mu+1)}{\Gamma(\mu-\alpha+1)}\left\{z^{\mu-\alpha}\right\}, \mu>-1 ; 0 \leq \alpha<1
$$

and

$$
I_{z}^{\alpha}\left\{z^{\mu}\right\}=\frac{\Gamma(\mu+1)}{\Gamma(\mu+\alpha+1)}\left\{z^{\mu+\alpha}\right\}, \mu>-1 ; \alpha>0 .
$$

Further properties of these operators with applications can be found in [2-6].

\section{PRELIMINARIES}

Let $\mathrm{H}$ be the class of functions analytic in the unit disk $U=\{z:|z|<1\}$ and for $a \in \mathrm{C}$ (set of complex numbers) and $n \in \mathrm{N}$ (set of natural numbers), let $\mathrm{H}[a, n]$ be the subclass of $\mathrm{H}$ consisting of functions of the form $f(z)=a+a_{n} z^{n}+a_{n+1} z^{n+1}+\cdots$. Let $\mathrm{A}$ be the class of functions $f$, analytic in $U$ and normalized by the conditions $f(0)=f^{\prime}(0)-1=0$. A function $f \in \mathrm{A}$ is called starlike of order $\mu$ if it satisfies the following inequality

$$
\mathfrak{R}\left\{\frac{z f^{\prime}(z)}{f(z)}\right\}>\mu,(z \in U)
$$

for some $0 \leq \mu<1$. We denoted this class $\mathrm{S}(\mu)$. A function $f \in \mathrm{A}$ is called convex of order $\mu$ if it satisfies the following inequality

$$
\mathfrak{R}\left\{\frac{z f^{\prime \prime}(z)}{f^{\prime}(z)}+1\right\}>\mu,(z \in U)
$$

for some $0 \leq \mu<1$. We denoted this class $\mathrm{C}(\mu)$. We note that $f \in \mathrm{C}(\mu)$ if and only if $z f^{\prime} \in \mathrm{S}(\mu)$. Furthermore, Let $\mathrm{P}$ be the subclass of analytic functions in the unit disk and take the formula

$$
p(z)=1+\sum_{n=1}^{\infty} a_{n} z^{n}, \quad \mathfrak{R}(p(z))>0, p(0)=1 .
$$

Let $f$ be analytic in $U$, g analytic and univalent in $U$ and $f(0)=g(0)$. Then, by the symbol $f(z) \prec g(z)$ ( $f$ subordinate to $g$ ) in $U$, we shall mean $f(U) \subset g(U)$.

Let $\phi: \mathrm{C}^{2} \rightarrow \mathrm{C}$ and let $h$ be univalent in $U$. If $p$ is analytic in $U$ satisfying the differential subordination $\left.\phi(p(z)), z p^{\prime}(z)\right) \prec h(z)$ then $p$ is called a solution of the differential subordination. The univalent function $q$ is called a dominant of the solutions of the differential subordination, $p \prec q$. If $p$ and $\left.\phi(p(z)), z p^{\prime}(z)\right)$ are univalent in $U$ and satisfy the differential superordination $\left.h(z) \prec \phi(p(z)), z p^{\prime}(z)\right)$ then $p$ is called a solution of the differential superordination. An analytic function $q$ is called subordinate of the solution of the differential superordination if $q \prec p$. For details (see [7]). 
Analogs to this definition, we impose the concept of fractional differential subordination.

Definition 2.1. Let $\varphi: C^{2} \rightarrow C$ and let $h$ be univalent in $U$. If $p$ is analytic in $U$ satisfying the fractional differential subordination $\varphi\left(p(z), z^{\alpha} D_{z}^{\alpha} p(z)\right) \prec h(z), 0 \leq \alpha<1$, then $p$ is called a solution of the fractional differential subordination. The univalent function $q$ is called a dominant of the solutions of the fractional differential subordination, $p \prec q$. If $p$ and $\varphi\left(p(z), z^{\alpha} D_{z}^{\alpha} p(z)\right)$ are univalent in $U$ and satisfy the fractional differential superordination $h(z) \prec \varphi\left(p(z), z^{\alpha} D_{z}^{\alpha} p(z)\right)$ then $p$ is called a solution of the fractional differential superordination. An analytic function $q$ is called subordinate of the solution of the fractional differential superordination if $q \prec p$.

It is clear that when $\alpha=0$, we have the differential subordination and the differential superordination of the first order. In the following sequel, we will assume that $h$ is an analytic convex function in $U$ with $h(0)=1$. For $0 \leq \alpha<1$, consider the fractional differential equation

$$
p(z)+\mu z^{\alpha} D_{z}^{\alpha} p(z)=g(z), \quad g(z) \prec h(z) .
$$

We will denote the class consisting of all solutions $p \in \mathrm{P}$ as $\mathrm{R}(\alpha, \mu, h)$, that is

$$
R(\alpha, \mu, h)=\left\{p \in P: p(z)+\mu z^{\alpha} D_{z}^{\alpha} p(z) \prec h(z), z \in U\right\} .
$$

Definition 2.2. [7] We denote by $Q$ the set of all functions $f(z)$ that are analytic and univalent on $\bar{U}-E(f)$ where $E(f):=\left\{\zeta \in \partial U: \lim _{z \rightarrow \zeta} f(z)=\infty\right\}$ and are such that $f^{\prime}(\zeta) \neq 0$ for $\zeta \in \partial U \backslash E(f)$.

\section{FRACTIONAL DIFFERENTIAL SUBORDINATION}

In this section, we establish some results which are related to the subordination of two functions. This will lead to develop and generalize the theory of differential subordinations. In addition, we show that the problem of finding best dominants of fractional subordination reduces to finding univalent solutions of fractional differential equations.

Theorem 3.1. Let $p(z)=a+a_{n} z^{n}+\ldots$ be analytic in $U$ with $p(z) \neq a$ and $n \geq 1$, and let $q \in Q$ with $q(0)=a$. If there exist points $z_{0} \in U$ and $\zeta_{0} \in \partial U \backslash E(q)$ such that $p\left(z_{0}\right)=q\left(\zeta_{0}\right) \quad$ and $\quad p\left(U_{r_{0}}\right) \subset q(U)$, where $\quad r_{0}=\left|z_{0}\right| \quad$ and $U_{r_{0}}:=\left\{z:|z|<r_{0}<1\right\}$, then there exists a positive real number $m \geq n$ such that

$$
z_{0}^{\alpha} D_{z_{0}}^{\alpha} p\left(z_{0}\right)=m \zeta_{0} \sum_{k=0}^{\infty}\left(\begin{array}{l}
\alpha \\
k
\end{array}\right) q^{(k)}\left(\zeta_{0}\right),
$$

where $\left(\begin{array}{l}\alpha \\ k\end{array}\right)$ is the normal binomial coefficients. 
Proof. Since $p$ is analytic in $U$, the set $p\left(\bar{U}_{r_{0}}\right)$ is bounded and $p\left(\bar{U}_{r_{0}}\right) \subset q(\bar{U}) \backslash E(q)$. By putting $f(z)=q^{-1}[p(z)]$, for $z \in \bar{U}_{r_{0}}$, then $f$ is analytic in $\bar{U}_{r_{0}}$ and satisfies $\left|f\left(z_{0}\right)\right|=\left|\zeta_{0}\right|=1, f(0)=0$ and $|f(z)| \leq 1$, for $|z| \leq r_{0}$. Furthermore, since $p(z)=q(\zeta)$, where $\zeta=f(z)$. In view of Remark 1.1, a computation yields

$$
\frac{z^{\alpha} D_{z}^{\alpha} f(z)}{f(z)}=z^{\alpha} o\left(z^{-\alpha}\right)=o(1):=m,
$$

where $m$ is a positive real number satisfying $m \geq n$. Consequently, by using Leibniz rule for fractional differentiation of analytic functions [8] yields

$$
\begin{aligned}
D_{z}^{\alpha} p(z) & =D_{z}^{\alpha}(q(\zeta)) \\
& =D_{z}^{\alpha}(q(f(z))) \\
& =\sum_{k=0}^{\infty}\left(\begin{array}{l}
\alpha \\
k
\end{array}\right) q^{(k)}(z) D_{z}^{\alpha-k} f(z) .
\end{aligned}
$$

Hence, by setting $z=z_{0}, \zeta=\zeta_{0}$, we obtain

$$
\begin{aligned}
z_{0}^{\alpha} D_{z_{0}}^{\alpha} p\left(z_{0}\right) & =\zeta_{0} \sum_{k=0}^{\infty}\left(\begin{array}{l}
\alpha \\
k
\end{array}\right) q^{(k)}\left(z_{0}\right) \frac{z_{0}^{\alpha} D_{z_{0}}^{\alpha-k} f\left(z_{0}\right)}{f\left(z_{0}\right)} \\
& =m \zeta_{0} \sum_{k=0}^{\infty}\left(\begin{array}{l}
\alpha \\
k
\end{array}\right) q^{(k)}\left(\zeta_{0}\right) .
\end{aligned}
$$

This completes the proof.

We next consider the subordination of two functions. This will lead to suggest the concept of the fractional differential subordinations.

Theorem 3.2. Let $p(z)=a+a_{n} z^{n}+\ldots$ be analytic in $U$ with $p(z) \neq a$ and $n \geq 1$, and let $q \in Q$ with $q(0)=a$. If $p$ is not subordination to $q$, then there exist points $z_{0}=r_{0} e^{i \theta_{0}} \in U$ and $\zeta_{0} \in \partial U \backslash E(q)$ for which $p\left(U_{r_{0}}\right) \subset q(U)$ and a real number $m$ such that

$$
\begin{aligned}
& \text { (i) } p\left(z_{0}\right)=q\left(\zeta_{0}\right) \\
& \text { (ii) } z_{0}^{\alpha} D_{z_{0}}^{\alpha} p\left(z_{0}\right)=m \zeta_{0} \sum_{k=0}^{\infty}\left(\begin{array}{l}
\alpha \\
k
\end{array}\right) q^{(k)}\left(\zeta_{0}\right),
\end{aligned}
$$

where $m$ is real.

Proof. Since $p(0)=q(0)$ and $p, q$ are analytic in $U$ we define

$$
r_{0}=\sup \left\{r: p\left(U_{r}\right) \subset q(U)\right\} \text {. }
$$

By the assumption $p(z)^{\circ} q(z)$ yields $p(U) \not \subset q(U)$. Since $p\left(\bar{U}_{r_{0}}\right) \subset \overline{q(U)}$, there exists $z_{0} \in \partial U_{r_{0}}$ such that $p\left(z_{0}\right) \in \partial q(U)$. Hence there exists $\zeta_{0} \in \partial U \backslash E(q)$ such that 
$p\left(z_{0}\right)=q\left(\zeta_{0}\right)$ and this completes conclusion (i). Conclusion (ii) follows by applying Theorem 3.1.

We shall define the class of generalized admissible functions. This class plays an important role in the theory of fractional differential subordinations. The proof follows by applying Theorem 3.2.

Definition 3.1. Let $\Omega$ be a set in $\mathrm{C}, q \in Q$ and $n$ be positive integer. The class of generalized admissible functions $\Psi_{n}[\Omega, q]$, consists of those functions $\psi: \mathrm{C}^{2} \times U \rightarrow \mathrm{C}$ that satisfy the admissible condition

$$
\psi\left(q(\zeta), m \zeta \sum_{k=0}^{\infty}\left(\begin{array}{l}
\alpha \\
k
\end{array}\right) q^{(k)}(\zeta) ; z\right) \notin \Omega,
$$

when $z \in U, \zeta \in \partial U \backslash E(q)$ and $m$ is real.

Note that when $\alpha=0$ and $m \geq n$ in Definition 3.1, we have the normal admissible functions.

Theorem 3.3. Let $\psi \in \Psi[\Omega, q]$ with $q(0)=a$. If $p(z)=a+a_{n} z^{n}+\ldots$ satisfies

$$
\psi\left(p(z), z^{\alpha} D_{z}^{\alpha} p(z) ; z\right) \in \Omega,
$$

then $p \prec q$.

Proof. Assume $p \nprec q$. By Theorem 3.2, there exists $z_{0}=r e^{i \theta} \in U$ and $\zeta_{0} \partial U \backslash E(q)$ and $m$ real that satisfy (i)-(ii). Thus by Definition 3.1, we have

$$
\psi\left(p\left(z_{0}\right), z_{0}^{\alpha} D_{z_{0}}^{\alpha} p\left(z_{0}\right) ; z_{0}\right) \notin \Omega
$$

which contradicts (2); hence $p \prec q$.

From the above result we pose dominants of fractional differential subordination (2) by using the generalized admissible function $\psi$.

Corollary 3.2. Let $q$ be univalent in $U$ with $q(0)=a$. And let $\Omega \subset \mathrm{C}, \psi \in \Psi\left[\Omega, q_{\rho}\right], \rho \in(0,1)$ with $q_{\rho}(z)=q(\rho z)$. If $p(z)=a+a_{n} z^{n}+\ldots$ satisfies (2) then $p \prec q$.

Proof. Since $q$ is univalent in $U$ then its univalent in $\bar{U}$; thus the set $E(q)=\varnothing$ and $q_{\rho} \in Q$. The class $\Psi\left[\Omega, q_{\rho}\right]$ is an admissible class ; therefore in view of theorem 3.3, $p \prec q_{\rho}$. But $q_{\rho} \prec q$, we deduce $p \prec q$.

Corollary 3.3. Let $\Omega \subset \mathrm{C}$ be simply connected domain and a conformal mapping $h: U \rightarrow \Omega$ such that $\Omega=h(U)$. And let $\psi \in \Psi[h(U), q]$ with $q(0)=a$. If $p(z)=a+a_{n} z^{n}+\ldots$ satisfies

$$
\psi\left(p(z), z^{\alpha} D_{z}^{\alpha} p(z) ; z\right) \prec h(z), \quad z \in U,
$$

then $p \prec q$.

Proof. Conditions (2) and (3) are equivalent. Thus in virtue of Theorem 3.3, we have $p \prec q$. 
Corollary 3.4. Let $h$ and $q$ be univalent in $U$, with $q(0)=a$ and set $q_{\rho}(z)=q(\rho z)$ and $h_{\rho}(z)=h(\rho z)$. Let $\psi: \mathrm{C}^{2} \times U \rightarrow \mathrm{C}$ satisfy

$$
\psi \in \Psi_{n}\left[h(U), q_{\rho}(U)\right], \quad \rho \in(0,1) .
$$

If $p(z)=a+a_{n} z^{n}+\ldots, \psi\left(p(z), z^{\alpha} D_{z}^{\alpha} p(z) ; z\right)$ is analytic in $U$ and

$$
\psi\left(p(z), z^{\alpha} D_{z}^{\alpha} p(z) ; z\right) \prec h(z), \quad z \in U
$$

then $p \prec q$.

Proof. By applying Theorem 3.3, we have $p \prec q_{\rho}$. But $q_{\rho} \prec q$, we deduce $p \prec q$.

Corollary 3.5. Let $h$ and $q$ be univalent in $U$, with $q(0)=a$ and set $q_{\rho}(z)=q(\rho z)$ and $h_{\rho}(z)=h(\rho z)$. Let $\psi: \mathrm{C}^{2} \times U \rightarrow \mathrm{C}$ satisfy

$$
\psi \in \Psi_{n}\left[h_{\rho}(U), q_{\rho}(U)\right], \quad \rho \in\left(\rho_{0}, 1\right), \rho_{0} \in(0,1) .
$$

If $p(z)=a+a_{n} z^{n}+\ldots, \psi\left(p(z), z^{\alpha} D_{z}^{\alpha} p(z) ; z\right)$ is analytic in $U$ and

$$
\psi\left(p(z), z^{\alpha} D_{z}^{\alpha} p(z) ; z\right) \prec h(z), \quad z \in U
$$

then $p \prec q$.

Proof. By applying Theorem 3.3, we have $p_{\rho} \prec q_{\rho}$. By letting $\rho \rightarrow 1^{-}$, we pose $p \prec q$.

Theorem 3.4. Let $h$ be univalent in $U$ and let $\psi: \mathrm{C}^{2} \times U \rightarrow \mathrm{C}$ such that the fractional differential equation

$$
\psi\left(q(z), z^{\alpha} D_{z}^{\alpha} q(z) ; z\right)=h(z)
$$

subject to the initial condition $q(0)=a$ has a univalent solution $q$ and one of the following conditions is satisfied

(i) $q \in$ Qand $\psi \in \Psi[h, q]$,

(ii) qisunivalentinUand $\psi \in \Psi\left[h, q_{\rho}\right], \quad \rho \in(0,1)$

(iii ) qisunivalentinUand $\psi \in \Psi\left[h_{\rho}, q_{\rho}\right], \quad \rho \in\left(\rho_{0}, 1\right), \rho_{0} \in(0,1)$.

If $p(z)=a+a_{n} z^{n}+\ldots, \psi\left(p(z), z^{\alpha} D_{z}^{\alpha} p(z) ; z\right)$ is analytic in $U$ satisfies (4) then $p \prec q$ and $q$ is the best dominant.

Proof. In view of Corollaries 3.3, 3.4 and 3.5, we have $q$ as the dominant of (4). Since $q$ is a solution of (5), it implies that $q$ is a solution for (4) and hence it is the best dominant.

\section{APPLICATIONS}

In this section, we deduce some applications of Theorem 3.3 and its corollaries. 
Theorem 4.1. Let $n$ be a positive integer, $\lambda>0$ and let $\mu_{0}$ be a solution for the equation

$$
\mu \pi=\frac{3 \pi}{2}-\tan ^{-1}\left[\sigma_{k}(\mu, \lambda, \alpha, n)\right], \quad k \geq 1,0 \leq \alpha<1 .
$$

In addition, let

$$
v=\mu+\frac{2}{\pi} \tan ^{-1}\left[\sigma_{k}(\mu, \lambda, \alpha, n)\right]
$$

where

$$
\begin{aligned}
\sigma_{k}(\mu, \lambda, \alpha, n):=1+ & \operatorname{in} \mu \lambda\left(\begin{array}{l}
\alpha \\
1
\end{array}\right) \frac{\left(1+r^{2}\right)}{2 r}+\mu(1-\mu)\left(\begin{array}{l}
\alpha \\
2
\end{array}\right)\left(\frac{n \lambda\left(1+r^{2}\right)}{2 r}\right)^{2}+\ldots \\
& +\left(\frac{-1}{i}\right)^{k}\left(\begin{array}{l}
\mu \\
k
\end{array}\right)\left(\begin{array}{l}
\alpha \\
k
\end{array}\right)\left(\frac{n \lambda\left(1+r^{2}\right)}{2 r}\right)^{k}
\end{aligned}
$$

for $0<\mu \leq \mu_{0}<1$. If $p \in \mathrm{H}[1, n]$, then for sufficient $\lambda>0$ and $\mu<1$

$$
p(z)+\lambda z^{\alpha} D_{z}^{\alpha} p(z) \prec\left[\frac{1+z}{1-z}\right]^{v} \Rightarrow p(z) \prec\left[\frac{1+z}{1-z}\right]^{\mu} .
$$

Proof. Let

$$
h(z)=\left[\frac{1+z}{1-z}\right]^{v} \text { and } \quad q(z)=\left[\frac{1+z}{1-z}\right]^{\mu},
$$

then the domains $h(U)$ and $q(U)$ are given by the sectors

$$
|\arg h|<\frac{v \pi}{2} \text { and }|\arg q|<\frac{\mu \pi}{2},
$$

respectively. We will assume two cases; the first case corresponds to a point on the boundary rays on the sectors $q(U)$ and the second case corresponds to corner of the sector. Assume that $p(z)^{\circ} q(z)$; therefore there exist points $z_{0}=r_{0} e^{i \theta_{0}} \in U$ and $\zeta_{0} \in \partial U$ for which $p\left(U_{r_{0}}\right) \subset q(U)$ such that $p\left(z_{0}\right)=q\left(\zeta_{0}\right)$.

If $p\left(z_{0}\right)=0$ and since $\mu<1$ implies that the sector angle of $q(U)$ is less than $\pi$ and $p\left(\left|z_{0}\right|\right)$ cannot pass through a corner without itself has a corner. Hence this case does not hold.

Consider the case when $p\left(z_{0}\right) \neq 0$. Assume that

$$
r i=\frac{1+\zeta_{0}}{1-\zeta_{0}}, \quad \zeta_{0} \neq \pm 1
$$

thus in view of Theorem 3.2 and for $k \geq 1$, we obtain 


$$
\begin{aligned}
& p\left(z_{0}\right)+\lambda z_{0}^{\alpha} D_{z_{0}}^{\alpha} p\left(z_{0}\right)=q\left(\zeta_{0}\right)+\lambda m \zeta_{0} \sum_{k=1}^{\infty}\left(\begin{array}{l}
\alpha \\
k
\end{array}\right) q^{(k)}\left(\zeta_{0}\right) \\
& =(r i)^{\mu}+\lambda m \zeta_{0} \sum_{k=1}^{\infty}\left(\begin{array}{l}
\alpha \\
k
\end{array}\right)\left[(r i)^{\mu}\right]^{(k)}\left(\zeta_{0}\right) \\
& =(r i)^{\mu}+\lambda m \zeta_{0}\left(\begin{array}{l}
\alpha \\
1
\end{array}\right)\left[(r i)^{\mu}\right]^{(1)}\left(\zeta_{0}\right)+\lambda m \zeta_{0}\left(\begin{array}{l}
\alpha \\
2
\end{array}\right)\left[(r i)^{\mu}\right]^{(2)}\left(\zeta_{0}\right)+\ldots \\
& =(r i)^{\mu}-m \lambda \mu\left(\begin{array}{l}
\alpha \\
1
\end{array}\right)(r i)^{\mu-1} \frac{\left(1+r^{2}\right)}{2}+\mu(\mu-1)\left(\begin{array}{l}
\alpha \\
2
\end{array}\right)(r i)^{\mu-2}\left[m \lambda \frac{\left(1+r^{2}\right)}{2}\right]^{2}-\ldots \\
& +(-1)^{k}\left(\begin{array}{l}
\mu \\
k
\end{array}\right)\left(\begin{array}{l}
\alpha \\
k
\end{array}\right)\left[m \lambda \frac{\left(1+r^{2}\right)}{2}\right]^{\mu} \\
& =(r i)^{\mu}\left[1+i m \mu \lambda\left(\begin{array}{l}
\alpha \\
1
\end{array}\right) \frac{\left(1+r^{2}\right)}{2 r}-\mu(\mu-1)\left(\begin{array}{l}
\alpha \\
2
\end{array}\right)\left(\frac{m \lambda\left(1+r^{2}\right)}{2 r}\right)^{2}+\ldots\right. \\
& \left.+\left(\frac{-1}{i}\right)^{k}\left(\begin{array}{l}
\mu \\
k
\end{array}\right)\left(\begin{array}{l}
\alpha \\
k
\end{array}\right)\left(\frac{m \lambda\left(1+r^{2}\right)}{2 r}\right)^{k}\right], \quad m \geq n \\
& =(r i)^{\mu}\left[1+i m \mu \lambda\left(\begin{array}{l}
\alpha \\
1
\end{array}\right) \frac{\left(1+r^{2}\right)}{2 r}+\mu(1-\mu)\left(\begin{array}{l}
\alpha \\
2
\end{array}\right)\left(\frac{m \lambda\left(1+r^{2}\right)}{2 r}\right)^{2}+\ldots\right. \\
& \left.+\left(\frac{-1}{i}\right)^{k}\left(\begin{array}{l}
\mu \\
k
\end{array}\right)\left(\begin{array}{l}
\alpha \\
k
\end{array}\right)\left(\frac{m \lambda\left(1+r^{2}\right)}{2 r}\right)^{k}\right], \quad m \geq n \\
& :=(r i)^{\mu} \sigma_{k}(\mu, \lambda, \alpha, m) \text {, }
\end{aligned}
$$

where $\sigma_{0}(\mu, \lambda, \alpha, m)=1$. This implies that

$$
\arg \left[p\left(z_{0}\right)+\lambda z_{0}^{\alpha} D_{z_{0}}^{\alpha} p\left(z_{0}\right)\right]=\frac{\mu \pi}{2}+\tan ^{-1}\left[\sigma_{k}(\mu, \lambda, \alpha, m)\right] .
$$

Since arctan is a increasing function and $m \geq n$ by choosing $0<\mu<1$ for all values $r \neq 0$ we impose

$$
\frac{\mu \pi}{2}+\tan ^{-1}\left[\sigma_{k}(\mu, \lambda, \alpha, n)\right] \leq \arg \left[p\left(z_{0}\right)+\lambda z_{0}^{\alpha} D_{z_{0}}^{\alpha} p\left(z_{0}\right)\right] \leq \frac{\mu \pi}{2}+\frac{\pi}{2} .
$$

By using condition (8), we have $\mu \leq 3-v$ for $0<\mu \leq \mu_{0}$ and consequently, we obtain

$$
\frac{v \pi}{2} \leq \arg \left[p\left(z_{0}\right)+\lambda z_{0}^{\alpha} D_{z_{0}}^{\alpha} p\left(z_{0}\right)\right] \leq 2 \pi-\frac{v \pi}{2} .
$$

This leads to $p\left(z_{0}\right)+\lambda z_{0}^{\alpha} D_{z_{0}}^{\alpha} p\left(z_{0}\right)$ lying outside the sector $h(U)$ which contradicts the assumptions of the theorem. This completes the proof.

Next we introduce some special cases of Theorem 4.1. When $\alpha \rightarrow 1$ and $\sigma_{k}(\mu, \lambda, \alpha, n)=n \lambda \mu$ in Theorem 4.1, we have the following result which can be found in [9]:

Corollary 4.1. Let $n$ be a positive integer, $\lambda>0$ and let $\mu_{0}$ be a solution for the equation

$$
\mu \pi=\frac{3 \pi}{2}-\tan ^{-1}[n \lambda \mu]
$$


In addition, let

$$
v=\mu+\frac{2}{\pi} \tan ^{-1}[n \lambda \mu],
$$

for $0<\mu \leq \mu_{0}$. If $p \in \mathrm{H}[1, n]$, then

$$
p(z)+\lambda z p^{\prime}(z) \prec\left[\frac{1+z}{1-z}\right]^{v} \Rightarrow p(z) \prec\left[\frac{1+z}{1-z}\right]^{\mu} .
$$

Example 4.1. By letting $n=\lambda=\alpha=v=k=1$, in Eq. (10), we obtain $\mu=0.64$ and

$$
p(z)+\lambda z p^{\prime}(z) \prec \frac{1+z}{1-z} \Rightarrow p(z) \prec\left[\frac{1+z}{1-z}\right]^{0.64} .
$$

Example 4.2. By putting $n=\lambda=v=k=1, \alpha=0.5$ in Eq. (7), we obtain $\mu=0.3$ and

$$
p(z)+\lambda z^{0.5} D_{z}^{0.5} p(z) \prec \frac{1+z}{1-z} \Rightarrow p(z) \prec\left[\frac{1+z}{1-z}\right]^{0.3} .
$$

Further studies in fractional differential subordination can be found in [10].

\section{ACKNOWLEDGMENTS}

The authors would like to thank the reviewers for their comments on earlier versions of this paper. This work is supported by LRGS/TD/2011/UKM/ICT/03/02.

\section{REFERENCES}

1. M. Darus, R.W. Ibrahim, Radius estimates of a subclass of univalent functions, Mathematical Vesnik 63, 155-58, 2011.

2. H.M. Srivastava, Y. Ling, G. Bao, Some distortion inequalities associated with the fractional derivatives of analytic and univalent functions, Journal of Inequalities in Pure and Applied Mathematics 2, 1-6, 2001.

3. H.M. Srivastava, S. Owa, Univalent Functions, Fractional Calculus, and Their Applications, Halsted Press, John Wiley and Sons, New York, Chichester, Brisban, and Toronto, 1989.

4. R.W. Ibrahim, M. Darus, Subordination and superordination for analytic functions involving fractional integral operator, Complex Variables and Elliptic Equations 53, 1021-1031, 2008.

5. R.W. Ibrahim, M. Darus, Subordination and superordination for univalent solutions for fractional differential equations, Journal of Mathematical Analysis and Applications 345, 871-879, 2008.

6. A. A. Kilbas, H. M. Srivastava and J.J. Trujillo, Theory and Applications of Fractional Differential Equations. North-Holland, Mathematics Studies, Elsevier 2006. 7. S.S. Miller and P.T. Mocanu, Differential Subordinations: Theory and Applications. Pure and Applied Mathematics No.225 Dekker, New York, 2000.

8. I. Podlubny, Fractional differential equations. San Diego: Academic Press; 1999.

9. S.S. Miller and P.T. Mocanu, Differential Subordinations and Univalent Functions, Michigan Mathematical Journal 28, 157-171, 1981.

10. R.W. Ibrahim, The concept of fractional differential subordination, Tamkang Journal of Mathematics 44, 53-60, 2013. 